\title{
Factores técnico-operativos y políticos determinantes de las decisiones de inversión privada en el sector confección zuliano
}

\author{
Sandrea, Maryana* \\ Boscán, Mariby* \\ Romero, Jenny* \\ Acosta, Ana*
}

\begin{abstract}
Resumen
Desde finales de la década de los setenta gran parte de las empresas venezolanas han experimentado un proceso de desinversión, mientras que otras sólo invierten lo necesario para sobrevivir. En este contexto, el objetivo de esta investigación consiste en determinar los factores técnicos-operativos y políticos que afectan las decisiones de inversión privada en el sector confección zuliano. El estudio realizado es descriptivo, incluyó una entrevista personal a 18 gerentes. Los resultados obtenidos muestran que la intención de actualización tecnológica local, la ubicación geográfica del estado y el funcionamiento de servicios públicos figuran como los factores técnico-operativos favorables a la decisión de inversión, mientras que como factores desfavorables destacan la actuación de asociaciones de apoyo a las empresas y el costo de los servicios públicos. Asimismo, destaca la influencia negativa de la situación política venezolana. Se concluye que la situación económica, política y la falta de apoyo institucional constituyen los factores de mayor relevancia para las decisiones de inversión privada en el sector.
\end{abstract}

Palabras clave: Inversión privada, factores técnico-operativos y políticos, sector confección.

Recibido: 05-11-29. Aceptado: 06-06-19

* Magíster en Gerencia de Empresas, Economistas. Docentes-Investigadoras de la Universidad del Zulia. Instituto de Investigaciones de la Facultad de Ciencias Económicas y Sociales. Departamento de Estudios de Microeconomía.

Email: marysandrea@yahoo.com - maribyboscán@yahoo.com - Jennyfrb@yahoo.com 


\title{
Technical-Operative and Political Factors that Determine Private Investment Decisions in the Zulia State Garment Sector
}

\begin{abstract}
Since the end of the nineteen seventies, a great number of Venezuelan companies have experienced a de-investment process, while others invested only in what was necessary to survive. In this context the objective of this research is to determine the technical-operating and political factors affecting private investment decisions in the Zulia State garment sector. The study is descriptive, and included personal interviews with 18 plant managers. The results obtained show: a) the intention to realize local technological up-dating, b) the geographical location of the state and the operation of public services figure as favorable technical-operating factors in the investment decision, and c) unfavorable factors were the performance of company support associations and the cost of public services. Also, the negative influence of the Venezuelan political situation is pointed out. The conclusion is that the economic and political situation and the lack of institutional support constitute the more relevant factors in private investment decisions in this sector.
\end{abstract}

Key words: Private investment, technical-operative and political factors, garment sector.

\section{Introducción}

A partir de finales de la década de los años setenta, específicamente en 1979 , se registra una marcada debilidad de la inversión privada venezolana, evidenciada en una fuerte caída de esta variable, la cual presenta leves recuperaciones en algunos periodos, y breve estabilidad en otros, pero en general muestra una tendencia decreciente, lo que implica, según Baptista (1997), una ruptura en el modo de acumulación que ha originado que muchas empresas experimenten un proceso de desinversión, mientras que otras sólo invierten lo necesario para sobrevivir. En este sentido, cabe destacar que para el año 2000 la Inversión Bruta Privada no alcanzó el $10 \%$ del Producto Interno Bruto (PIB) (BCV, 2000-2001), además a lo largo de las últimas dos décadas han dejado de realizarse inversiones necesarias en áreas vitales para el desarrollo empresarial (maquinarias y equipos, capacitación del recurso humano e infraestructura); al mismo tiempo muchas empresas redujeron las dimensiones de sus negocios o bien cerraron.

Esta situación revela la presencia de importantes obstáculos a la inversión privada, entre los que se encuentran: Inestabilidad macroeconómica y política, carencia de opciones de financiamiento (altos costos, plazo y trámites inadecuados a las necesidades de las empresas), factores de orden técnico-operativos que dificultan la materialización de la inversión privada, falta de apoyo institucional, rol del Estado empresario, que se ha reservado para sí las áreas de mayor rentabilidad en la economía venezolana, así como una visión localista y corto placista del negocio por parte de los empresarios (Sandrea, 2005).

La inversión privada dirigida a la actualización de maquinarias, equipos y 
Factores técnico-operativos y políticos determinantes de las decisiones de inversión Sandrea, Maryana; Boscán, Mariby; Romero, Jenny y Acosta, Ana

tecnología constituiría una excelente vía para alcanzar el crecimiento del sector confección, pues la misma permitiría la reducción de los costos de producción, así como un mejoramiento de la calidad del producto, cuestión que redundará en un incremento de la competitividad. De igual forma, es importante considerar otros factores como los de carácter político (estabilidad y confianza en el sistema político venezolano) y su incidencia en las decisiones de inversión de los empresarios. En tal sentido, el presente estudio tiene como propósito determinar los factores técnicos-operativos y políticos que afectan la decisión de inversión privada en las empresas de reducido tamaño del sector confección en el municipio Maracaibo del estado Zulia.

En relación a la metodología se utilizó un diseño no experimental, transversal de campo, dado que la información relativa a la variable factores técnico-operativos y políticos determinantes de la inversión privada, se obtuvo en un momento específico de tiempo (marzo-abril 2005). El estudio presentado es descriptivo, porque señala la opinión de los gerentes del sector confección sobre los referidos factores en relación a sus decisiones de inversión. Así mismo, la naturaleza de la investigación exigió la utilización de fuentes de información primaria, representadas por el uso de la entrevista personal guiada con un formato semiestructurado.

La población entrevistada está representada por los dueños-gerentes de empresas de reducido tamaño que confeccionan todo tipo de ropa, ubicadas geográficamente (al menos su área de producción) en el municipio Maracaibo del estado Zulia. En este sentido, cabe destacar que, según las estadísticas proporcionadas por la base de datos del Programa "Factores de competitividad de las pequeñas y medianas empresas (PYMES) manufactureras de la región Zuliana (2004)", del Instituto de Investigaciones de la Facultad de Ciencias Económicas y Sociales de la Universidad del Zulia, existen en el municipio Maracaibo del estado Zulia un total de sesenta y dos (62) empresas pertenecientes al sector confección. Sin embargo luego de realizar un censo de estas empresas se encontró que: Once (11) no forman parte del estudio por ser sólo revendedores y no fabricantes, trece (13) estaban cerradas o no pudieron ser localizadas, y otras 20 no desearon brindar información para el estudio, por lo tanto los resultado de la investigación se refieren sólo a dieciocho (18) empresas del sector confección.

\section{Evolución de la inversión privada en el ámbito nacional durante el período 1970-2003}

La inversión se refiere a la asignación de fondos (desembolso de recursos financieros) para la adquisición, creación, mejora y mantenimiento de activos circulantes, fijos (tangibles e intangibles) así como de la infraestructura de apoyo a la producción, que se realizan con la expectativa de obtener un retorno en un tiempo futuro (Sandrea, 2005). A fin de simplificar el análisis sobre este punto se seleccionó como indicador fundamental la Inversión Bruta Fija (IBF), como porcentaje del Producto Interno Bruto (PIB).

La Inversión Bruta Fija se refiere al gasto en bienes de capital fijos (medios de producción cuya duración física es 
más o menos larga, más de un año), sin considerar el gasto en variación de existencias, incluido en la Inversión Bruta. Son ejemplos de inversiones fijas la compra de nuevas maquinarias y equipos, vehículos de transporte, obras de infraestructura, entre otras. Este tipo de inversión se asocia con el crecimiento de la capacidad productiva (Baptista, 2004).

En los últimos veinte años la Inversión Bruta Fija en Venezuela ha mostrado una tendencia decreciente, pudiendo ser calificada en general como insuficiente e inestable. El análisis de la inversión en activos fijos en Venezuela, como porcentaje del PIB, muestra un importante crecimiento desde 1970 hasta 1978, producto de las masivas inversiones relacionadas con la actividad petrolera (especialmente a partir de la nacionalización de la industria); luego a partir de 1979 y hasta 1983 ocurre una fase de decrecimiento evidenciada por una caída sostenida de tal mag- nitud que deja a la Inversión Bruta Fija en niveles cercanos a los de 1971 (ver Figura 1). Lo que revela que el ritmo de crecimiento de la inversión llevada a cabo en el periodo anterior era insostenible, así mismo en esta época se produce una fuerte devaluación de la moneda venezolana, lo que contribuyó a frenar las inversiones en el país.

Posteriormente, se presenta un periodo de gran variabilidad desde 1984 hasta 2001 caracterizado por subidas y bajadas considerables, aun cuando de 1990 a 1992 se observa un aumento importante en la variable esta no llega a compararse con los niveles alcanzados en la segunda mitad de la década de los setenta, mientras que durante los años 2002 y 2003 experimenta una fuerte caída alcanzando su nivel histórico más bajo $(8,50 \%$ del PIB) desde 1970 (ver Figura 1).

Este comportamiento se mantiene, aún más acentuado, en el caso del com-

\section{Figura 1. Participación porcentual de la Inversión Bruta Fija sobre el Producto Interno Bruto (Base de datos en millones de bolívares a precios de 1984)}

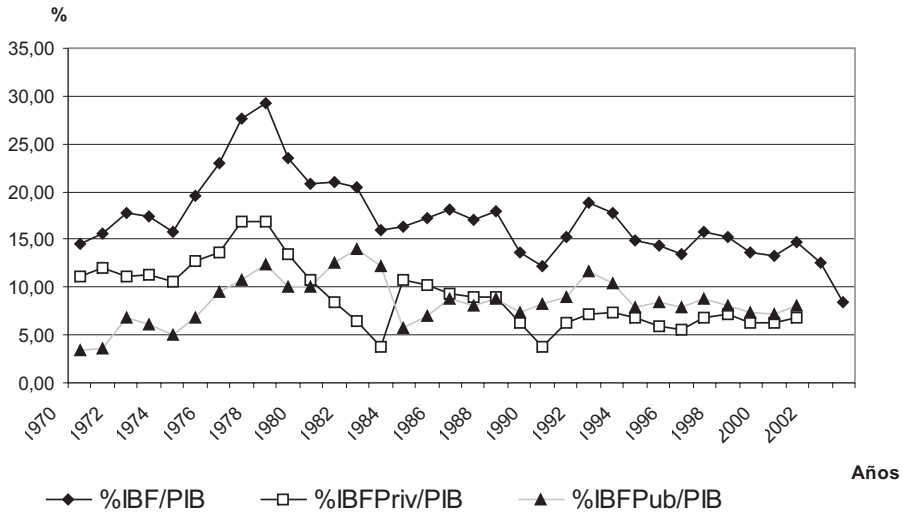

Fuente: Sandrea, 2005. Basado en los datos de: Baptista, 1997a; Anuarios de Cuentas Nacionales BCV, 1991; 1994; 1999; 2000-2001. Informes económicos BCV 2003 y 2004. 
Factores técnico-operativos y políticos determinantes de las decisiones de inversión Sandrea, Maryana; Boscán, Mariby; Romero, Jenny y Acosta, Ana

ponente privado (IBFPriv), el cual cae sostenidamente desde 1978 hasta 1983, mientras que en la inversión bruta pública en activos fijos (IBFPub) se evidencia una tendencia a superar la inversión privada. Durante la década de los noventa se muestra una leve preponderancia de la IBFPub sobre la privada, en tanto que la iniciativa privada perdió el papel destacable que reflejó entre los años cincuenta hasta finales de los setenta (Parra, 2001). Hasta 1978, la formación de capital en el sector manufacturero experimentó una etapa de rápida expansión de nuevas inversiones privadas, con fines de ampliación, renovación e instalación de plantas industriales (García y Plaza, 2000). Sin embargo, posterior al referido año, la iniciativa privada se basó sólo en la amortización de las inversiones pasadas, lo que según Baptista (1997) se explica porque ocurre una ruptura en el modo de acumulación venezolano.

Según los datos recabados por Penfold (2002), un comportamiento similar al de la inversión bruta fija como porcentaje del PIB, se registra en la inversión privada per cápita a precios de 1984, sin embargo, en este indicador la problemática es aún más acentuada, pues la población ha seguido su comportamiento ascendente. La tendencia decreciente de la inversión privada per cápita ha sido tan persistente que para el año 2000 está prácticamente a un nivel similar al que se produjo hace cincuenta y cinco (55) años atrás.

Esta evolución desfavorable de la inversión privada ha sido, según Penfold (2002), una de las causas de la crisis económica, política y social venezolana. Por lo tanto, la reactivación de la inversión se- ría un factor clave para disminuir el desempleo y la pobreza, así como lograr mejoras significativas en salud y educación. Para lo cual sería necesario analizar los factores determinantes de las decisiones de inversión de los empresarios, dentro de los cuales, dada la delimitación del estudio, se tratarán los aspectos técnicooperativos y políticos.

\section{Factores técnicos- operativos y políticos determinantes en la inversión privada}

Los factores técnico-operativos incluyen adaptación e incorporación de avances tecnológicos, requerimientos de calidad y disponibilidad de materias primas y materiales, transporte y aprovisionamiento, localización y tamaño óptimo de la planta e ingeniería del proyecto. Dentro de este marco de ideas para el presente estudio se analizarán los siguientes: Factor tecnológico, factor geográfico, y factor plataforma de apoyo al proceso productivo: que incluye seguridad física de bienes y personas, infraestructura y servicios, así como el rol de las instituciones que respaldan la actividad empresarial.

Según McConnell y Brue (2001) el factor tecnológico afecta las decisiones de inversión. El rápido surgimiento de innovaciones genera la idea de incorporar los adelantos técnicos al proceso productivo, debido a que pueden provocar aumento de los ingresos por ventas y/o reducción en los costos, lo cual redunda en un incremento del retorno esperado. Sin embargo, con relación a este factor aspectos como la adaptabilidad de la tecno- 
logía, el costo de adquisición y la velocidad de obsolescencia técnica pueden afectar la decisión de inversión.

Para Shatz (2001), las características geográficas pueden tener un peso importante en la selección de la localidad donde se decida materializar una inversión, pues la cercanía a zonas costeras y pobladas facilita la comercialización internacional.

Al mismo tiempo, la seguridad física de personas y bienes involucrados en los proyectos constituye un aspecto que incide en la decisión de inversión. "Los altos índices de actividades delictivas desalientan las inversiones, modificando la forma de funcionamiento de los negocios, pues elevan el riesgo percibido por los dueños del capital" (Monteferrante, 2002: 21).

Tal como lo señalan autores como Porter (1991), Penfold (2002) y Malavé (2002), una buena infraestructura de servicios de apoyo estimula la inversión, siendo en este sentido el servicio eléctrico uno de los principales, cuya disponibilidad y confiabilidad incide en la decisión de invertir, especialmente para las empresas manufactureras.

Por otro lado, en cuanto a los aspectos políticos, cabe destacar que dentro de éstos se incluyen el impacto del riesgo político dentro de la decisión de inversión (influencia de la percepción de estabilidad del sistema político sobre las decisiones de inversión), la firmeza y capacidad de ajuste del marco jurídico vigente y otras regulaciones al proceso inversor.

Según Penfold (2002), la estabilidad del sistema político cobra especial importancia en el caso de economías volátiles y vulnerables a los cambios de los precios en los mercados internacionales, como lo es la venezolana. Las características rentista y volátil de ésta economía implican que el riesgo político evidencia un componente estructural.

El comportamiento estatal afecta directa e indirectamente a las inversiones en términos de garantías sobre derechos de propiedad, derechos contractuales, que pudieran constituirse en un obstáculo a la inversión privada (Cohen y Sabal, 1988). Otro aspecto importante se refiere a la conveniencia del marco legal vigente para proteger los intereses de las organizaciones privadas, así como la actuación del sistema judicial en la solución de conflictos presentados en la actividad productiva (tiempo de respuesta, costos e imparcialidad).

\section{Determinantes de la decisión de inversión privada en el sector confección zuliano}

El sector confección comprende las empresas dedicadas al diseño, corte y costura de tela y otros materiales provistos por la industria textil, hasta dejarlos listos para ser usados por el consumidor final como indumentaria de vestir, exceptuando al calzado (Romero et al, 2000). En la actualidad a nivel nacional, este sector presenta una serie de problemas entre los que resaltan: Alto grado de fragmentación de la oferta e informalidad, debilidad crónica en la demanda interna, importantes niveles de capacidad ociosa, cercanos al 50\% (Morales et al, 2002), altos costos de materias primas e importante rezago tecnológico, problemática que también existe en el estado Zulia, destacando además que esta no es la zona de mayor concentración de empresas dedi- 
Factores técnico-operativos y políticos determinantes de las decisiones de inversión Sandrea, Maryana; Boscán, Mariby; Romero, Jenny y Acosta, Ana

cadas a la confección de ropa (región capital), por tanto las iniciativas para el impulso del sector a nivel nacional no han incluido tradicionalmente al estado Zulia (Sandrea y Boscán, 2004).

Según el criterio utilizado en el Decreto Ley para la Promoción y Desarrollo de la Pequeña y Mediana Industria en el país (República Bolivariana de Venezuela, 2001), las firmas que conforman el sector confección marabino son fundamentalmente micro y pequeñas empresas, cabe destacar que no se encontraron medianas empresas en el estudio realizado. El referido criterio, considera dos variables para la clasificación de industrias: Número de empleados y volumen de ventas en unidades tributarias.

En este sentido, para marzo-abril del 2005 un 77,78\% de las organizaciones consideradas se clasificaron como micro empresas, ya que poseen menos de 10 empleados, mientras que el restante $22,22 \%$ resultaron pequeñas empresas, pues emplean entre 11 y 50 personas. De acuerdo a este criterio ninguna de las empresas censadas clasifica como mediana. Con respecto a las ventas totales anuales en Unidades Tributarias (UT), éste no pudo ser tomado como indicador del tamaño de la empresa ya que un $44,44 \%$ de los gerentes-dueños entrevistados no respondieron a éste reactivo, en tanto que el resto afirmó que las ventas anuales eran entre 0 a 9000 UT, lo que cataloga a sus organizaciones como microempresas (Ver Tabla 1).

Comparando el criterio contenido en el Decreto-Ley para la Promoción de la Pequeña y Mediana Industria y el que todavía utiliza el Instituto Nacional de Estadísticas (INE), se tiene que, éste nuevo criterio introduce la innovación del uso de las ventas en unidades tributarias como variable clasificatoria, sin embargo su aplicación aún no se ha extendido a nivel nacional, además presenta el inconveniente de que muchas empresas consideran que el nivel de las ventas en unidades monetarias es confidencial. Adicionalmente, amplía el rango del número de empleados considerados como límite de la clasificación para la pequeña industria y lo reduce para la mediana, a la par que elimina la distinción entre mediana superior e inferior.

La flexibilidad en el tamaño de las empresas del sector confección les permite entrar y salir libremente del merca-

Tabla 1

Clasificación de las empresas del sector confección zuliano atendiendo al número de empleados.

\begin{tabular}{lcc}
\hline Tamaño de la empresa & No. Empresas & $\%$ \\
\hline Micro empresa (entre 0 y 10 empleados) & 14 & 77,78 \\
Pequeña industria (entre 11 y 50 empleados) & 4 & 22,22 \\
Mediana Industria (entre 51 y 100 empleados) & 0 & 0,00 \\
Gran industria (más de 100 empleados) & 0 & 0,00 \\
Total & 18 & 100,00 \\
\hline
\end{tabular}

Fuente: Sandrea, 2005. Entrevista a los empresarios del sector confección. 
do, por lo que es muy factible el cierre temporal, cambios de nombre, ubicación, entre otros. Lo anterior tiene su explicación en la profundización de la crisis económica venezolana que ha contribuido a la reducción del tamaño y/o cierre de muchas de las empresas confeccionistas, en este sentido, el sector ha pasado de estar conformado principalmente por pequeñas y medianas empresas a estar compuesto mayoritariamente por micro y pequeñas empresas. En términos de la permanencia de estas empresas en el mercado marabino, una gran parte de las mismas $(66,67 \%)$ son relativamente jóvenes (menos de 10 años), aunque para algunas lo realmente nuevo es el cambio de razón social y no la experiencia en el negocio.

La industria de la confección zuliana fabrica una variedad de productos tales como franelas, uniformes, ropa para niños, camisas para caballeros, vestidos, entre otros, siendo los uniformes $(27,78 \%)$ para marzo-abril de 2005 el producto líder (en aceptación y ventas) con mayor frecuencia relativa, mientras que para 1999 su producto líder eran las franelas (Morales et al, 2002). Es posible que el desplazamiento del producto franelas por uniformes, se deba a la profundización de la orientación localista del sector, por lo que muchos de estos establecimientos se dedican a fabricar uniformes para organizaciones públicas y privadas, colegios y otros institutos educativos.

Por otro lado, dentro de la problemática que afecta al sector destaca un importante rezago tecnológico, así como altos niveles de capacidad ociosa, cercanos al $50 \%$ (Morales et al, 2002), por lo que el incremento de la inversión en tecnología de punta y capital de trabajo resulta un factor clave para superar la crisis del sector. En este sentido, según la mayoría de los entrevistados $(77,78 \%)$ los cambios tecnológicos realizados especialmente por sus competidores locales, impulsan su decisión de inversión, sin embargo, dadas las dimensiones del atraso tecnológico que presentan estas empresas, la relación cambios tecnológicos e inversión se queda sólo en la intención y pocas veces se concreta en comportamientos que impliquen la incorporación de importantes adelantos técnicos (ver Figura 2).

En general puede decirse que el $61,11 \%$ de los gerentes-dueños entrevistados reconocieron que los activos de sus empresas presentan obsolescencia técnica en algún grado. De éste porcentaje, un $27,78 \%$ consideró que el nivel de obsolescencia técnica de los activos de la empresa oscilaba entre uno a cinco años, un $22,22 \%$ manifestaron que éste iba de seis a diez años, mientras que un $11,11 \%$ de los gerentes consultados indicó que el nivel de obsolescencia técnica de los activos de su empresa era superior a diez años. Mientras que el restante 38,89\% de los entrevistados revelaron que los activos de sus empresas no presentan obsolescencia técnica en comparación con otros competidores locales (ver Figura 3).

Las empresas del sector confección zuliano se encuentran atrapadas en una difícil paradoja, por un lado no pueden acceder a mercados internacionales debido a que sus volúmenes de producción son limitados, al tiempo que presentan niveles importantes de capacidad ociosa, debido entre otras causas a la debilidad de la demanda interna. Por lo que no se sienten motivados a ampliar la ca- 
Factores técnico-operativos y políticos determinantes de las decisiones de inversión Sandrea, Maryana; Boscán, Mariby; Romero, Jenny y Acosta, Ana

Figura 2

Cambios Tecnológicos como factor que impulsa la decisión de inversión privada

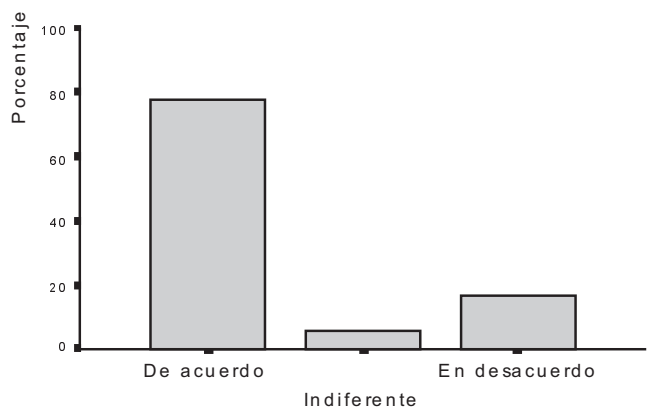

Alternativas de respuesta

Fuente: Sandrea, 2005. Con base a la entrevista realizada a empresarios del sector confección.

\section{Figura 3}

Nivel de obsolescencia técnica

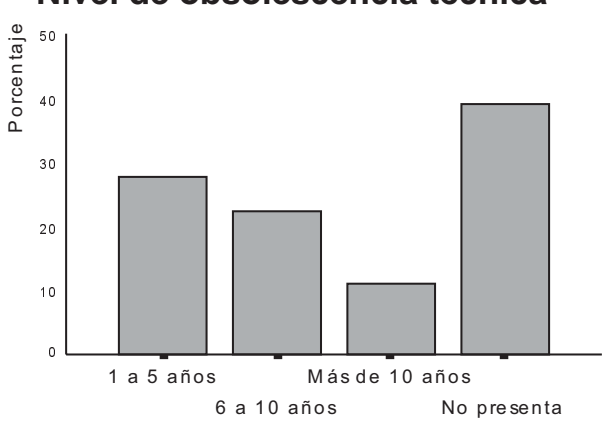

Nivel de obsolecencia técnica

Fuente: Sandrea, 2005. Con base a la entrevista realizada a empresarios del sector confección.

pacidad productiva, adicionalmente la visión localista del negocio, y la desactualización tecnológica en comparación con estándares internacionales complican aún más la situación.

Otro de los factores que teóricamente afectan las decisiones de inversión se refiere a la ubicación geográfica de la localidad, a este respecto la mayor parte de los entrevistados $(61,11 \%)$ estuvo de acuerdo en que las características geográficas del estado Zulia (Ubicación y cercanía al mercado colombiano, entre otros) constituyen un estímulo a la inversión privada, dado que algunos insumos utilizados (especialmente la tela) son importados desde Colombia, la proximidad con dicho mercado textil abarata el costo de materias primas y facilita su obtención; aunque señalaron que éste no es un factor primordial a la hora de tomar decisiones de inversión (ver Figura 4). 
Figura 4

Características geográficas de la región como factor que impulsa la inversión privada

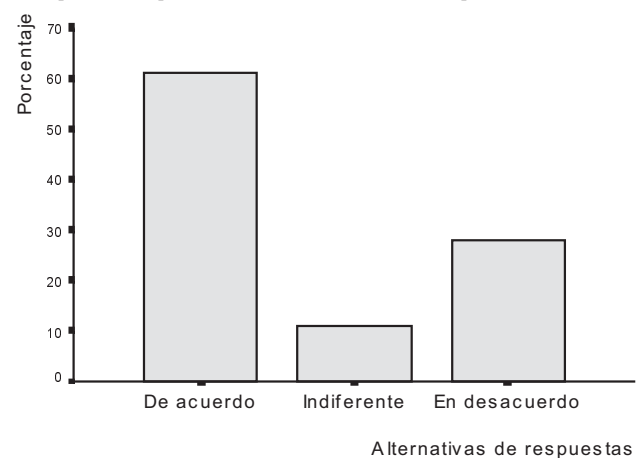

Fuente: Sandrea, 2005. Con base a la entrevista realizada a empresarios del sector confección.

Igualmente, la seguridad física de personas y bienes involucrados en los proyectos de inversión, constituye otro factor importante en las decisiones gerenciales. De acuerdo a los resultados obtenidos en las entrevistas realizadas a los empresarios del sector confección, un $50 \%$ de éstos consideraron que las actividades delictivas (hurto de materiales de trabajo, atracos, y otros) en la región zuliana han frenado las decisiones de inversión en sus empresas, sin embargo un porcentaje muy cercano $(44,44 \%)$ señaló estar en desacuerdo con tal apreciación, aclarando que las condiciones de seguridad física han cambiado ciertos manejos operativos en el negocio, pero no frenan en forma importante las decisiones de inversión, mientras que el resto fue indiferente ante tal aspecto. Por tanto, en este particular no es posible observar una tendencia de respuesta clara por parte de los entrevistados (ver Figura 5).

Adicionalmente, entre los factores operativos relevantes para las decisiones de inversión, se encuentran las condicio- nes de la plataforma de apoyo al proceso productivo. Venezuela tradicionalmente, "ha sido uno de los países con mejor dotación de infraestructura en América Latina..." (Singer y Lombardi, 2002: 35). Sin embargo, de acuerdo a la opinión de los citados autores, la inversión pública en infraestructura y servicios ha sido insuficiente en las últimas décadas, no se han realizado las inversiones de reposición y el mantenimiento apropiado a las obras físicas, redundando en una disminución de la calidad de los servicios e infraestructura de apoyo a las actividades productivas.

En términos de la entrevista realizada a los empresarios del sector confección, pudo constatarse que la mayoría de ellos $(55,56 \%)$ están de acuerdo en que las condiciones de infraestructura física de la plataforma operativa del país, y en particular la del estado Zulia, constituye un estímulo importante para las decisiones de inversión (ver Figura 6), sobre todo en cuanto al acceso a puertos (puerto de Maracaibo), aeropuertos (aeropuer- 
Factores técnico-operativos y políticos determinantes de las decisiones de inversión Sandrea, Maryana; Boscán, Mariby; Romero, Jenny y Acosta, Ana

Figura 5

Inseguridad física de personas y bienes como obstáculo a la inversión privada

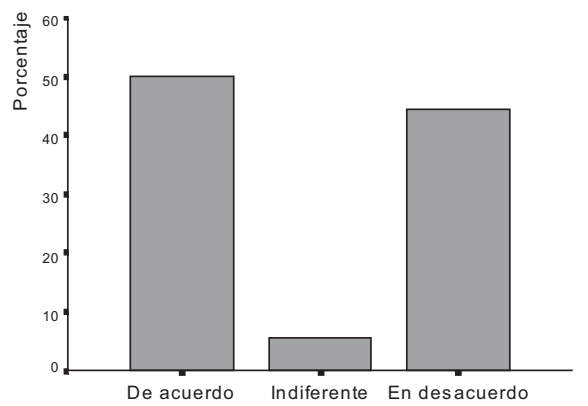

Alternativas de respuesta

Fuente: Sandrea, 2005. Con base a la entrevista realizada a empresarios del sector confección.

Figura 6

Plataforma operativa como estímulo a la inversión privada

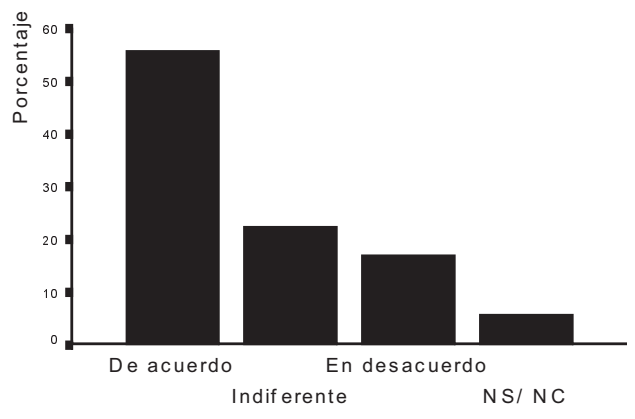

Alternativ as de respuesta

Fuente: Sandrea, 2005. Con base a la entrevista realizada a empresarios del sector confección.

to internacional la Chinita), así como una red de carreteras en buen estado (puente sobre el lago de Maracaibo, carretera Lara-Zulia; Falcón-Zulia, entre otras).

Asimismo, en cuanto al funcionamiento de los servicios públicos, la provisión deficiente de los mismos ha afectado el crecimiento económico y la competitividad del país. Siguiendo el estudio realizado por el Consejo Nacional para la Promoción de la Inversión Privada (CONA-
PRI) en el año 2002 (citado por Malavé, 2002), se pudo constatar que el servicio de apoyo a nivel nacional que más fallas presenta (medidas en número de días sin servicio) es el del suministro de agua (en la mayoría de los sectores productivos), siendo la ciudad de Maracaibo el lugar donde se ven afectadas un mayor número de empresas. Dentro del sector confección las fallas en el suministro de agua afecta directamente la fase de limpieza 
(lavado de algunos tipos de ropa), estampado y teñido de telas e indirectamente a todas las fases, pues incide sobre la productividad que el trabajador puede generar, al ser este un servicio básico.

En relación a la entrevista realizada a los empresarios del sector confección, pudo constatarse que el funcionamiento de los servicios público (agua, gas, electricidad y comunicaciones), fue considerado como favorable para la ejecución de nuevas inversiones por la mayoría de los entrevistados $(61,11 \%)$, sin embargo igual porcentaje afirmó que el costo de éstos servicios constituye un freno importante a las decisiones de inversión (ver Figura 7 y 8).

Con respecto a los servicios aduanales una gran parte de los entrevistados estuvo de acuerdo en que este tipo de trámites constituyen un obstáculo importante para las decisiones de inversión en su empresa $(44,44 \%)$ debido a que utilizan materias primas (tela) importada y al no contar con este insumo en tiempo optimo sus operaciones se ven afectadas negati- vamente (fase de compra y recepción de materias primas e insumos), a pesar de que la totalidad de los entrevistados manifestaron no realizar directamente importaciones de materias primas, sino que los bienes e insumos de origen foráneo que utilizan en sus procesos productivos los adquieren a través de intermediarios que realizan las funciones de importadores directos, sin embargo consideraron que los trámites aduanales (documentación exigida y tiempo empleado para la entrega de la mercancía al importador) constituyen la principal causa del retardo en la obtención de telas importadas (ver Figura 9).

Asimismo, la actuación de las cámaras y otras asociaciones privadas de apoyo a las empresas no ha favorecido la ejecución de inversiones, según la opinión de un 72,22\% de los entrevistados (ver Figura 10), quienes estuvieron en desacuerdo con el planteamiento de que el comportamiento de éste tipo de instituciones alguna vez haya impulsado las inversiones en el sector, muy por el contrario,

Figura 7

Funcionamiento de servicios públicos como factor de estímulo a la inversión privada

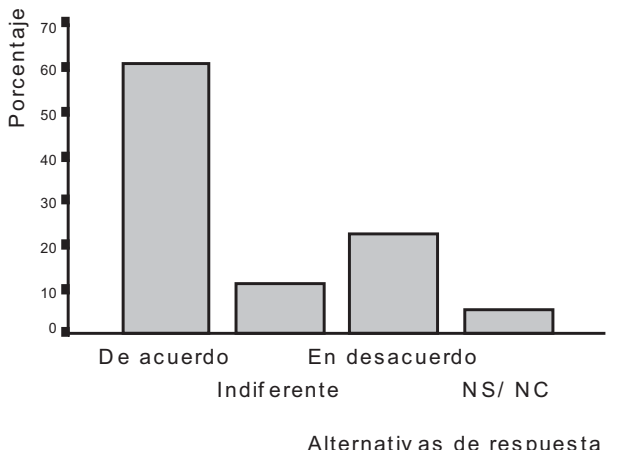

Fuente: Sandrea, 2005. Con base a la entrevista realizada a empresarios del sector confección. 
Factores técnico-operativos y políticos determinantes de las decisiones de inversión Sandrea, Maryana; Boscán, Mariby; Romero, Jenny y Acosta, Ana

Figura 8

Costo de los servicios públicos como obstáculo a la inversión privada

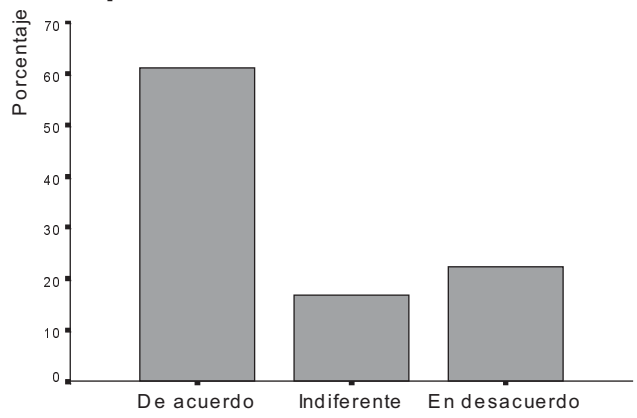

Alternativas de respuesta

Fuente: Sandrea, 2005. Con base a la entrevista realizada a empresarios del sector confección.

Figura 9

Servicios aduanales como obstáculos a la inversión privada

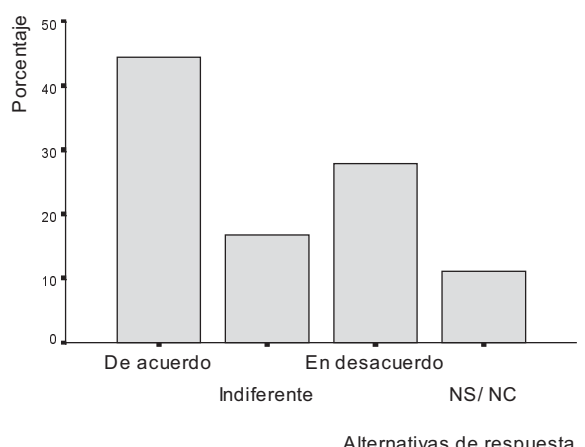

Fuente: Sandrea, 2005. Con base a la entrevista realizada a empresarios del sector confección.

Figura 10

Actuación de agrupaciones empresariales como estímulo a la inversión privada

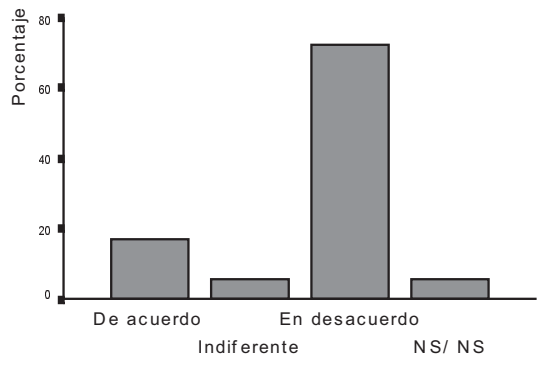

Alternativ as de respuesta

Fuente: Sandrea, 2005. Con base a la entrevista realizada a empresarios del sector confección. 
la mayor parte de los dueños-gerentes consultados manifestaron no pertenecer a ninguna cámara u asociación empresarial debido a que según su opinión, éstas organizaciones no representaban un apoyo real para las empresas.

En otro orden de ideas, uno de los elementos que podría ser considerado como determinante en las decisiones de inversión está referido al factor político, por cuanto en los últimos años en el país han ocurrido hechos que permiten catalogarlo como políticamente inestable, entre los cuales pueden mencionarse: La insurrección social del 27 y 28 de febrero de 1989, la sublevación militar del 4 de febrero de 1992, la salida anticipada de Carlos Andrés Pérez (presidente constitucional para el periodo 1989-1994), cambios políticos caracterizados por modificaciones del sistema, reglas y actores (se creó una nueva constitución, el sistema político pasó de estar centrado en "partidos" a centrarse en el Presidente de la República), los sucesos del 11 de abril del año 2002, el paro empresarial de finales del 2002 y principio del 2003 (Penfold, 2002). Los cambios en el sistema político generalmente generan incertidumbre en los ámbitos empresariales e incrementan el riesgo político del país; luego, mayor riesgo exige un mayor retorno financiero, por lo tanto sólo se desarrollaran proyectos de inversión extremadamente rentables.

Según estudios del Banco Mundial sobre el desarrollo económico 20002001, Venezuela destaca entre los países de América Latina que poseen mayores obstáculos a los negocios (elevados costos de transacción: obtención de permisos, licencias, gastos en seguridad personal y de las instalaciones, etc.) (Banco Mundial, 2003).

Por otro lado, de acuerdo a la opinión de la mayoría $(55,56 \%)$ de los dueños-gerentes de las empresas del sector confección zuliano entrevistados, la situación política venezolana desmotiva su deseo de invertir, fundamentalmente se refirieron a las condiciones generales de incertidumbre, y desconfianza en la política venezolana, así mismo, muchos recordaron las repercusiones negativas del paro cívico de finales del año 2002 (cierre de empresas o redimensionamiento del tamaño de otras) (ver Figura 11).

En cuanto al marco jurídico-institucional venezolano, Penfold (2002: 12) considera que existe una débil protección a los derechos de propiedad (especialmente en el caso de invasión de terrenos e inmuebles, donde la actuación de las autoridades competentes no es diligente, el proceso para la solución del conflicto es lento o simplemente no puede resolverse en un tiempo determinado), por lo que el funcionamiento del Estado de Derecho es percibido como uno de los principales obstáculos a la inversión. "La incertidumbre político-institucional es uno de los factores que mejor explican la caída de la inversión privada en Venezuela y otros países de América Latina". En la medida en que la intervención gubernamental genere incertidumbre sobre los derechos de propiedad, el riesgo operativo aumenta y por lo tanto, se eleva el nivel de ganancia que se debe ofrecer a los inversionistas para que estén dispuestos a arriesgar su capital (Cohen y Sabal, 1988).

Puede decirse que el sistema de derecho mercantil venezolano es lento, 
Factores técnico-operativos y políticos determinantes de las decisiones de inversión Sandrea, Maryana; Boscán, Mariby; Romero, Jenny y Acosta, Ana

\section{Figura 11}

\section{Situación política como obstáculo a la decisión de inversión}

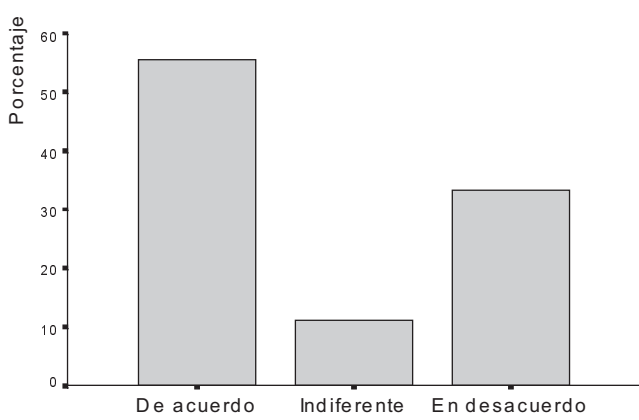

Alternativas de respuesta

Fuente: Sandrea, 2005. Con base a la entrevista realizada a empresarios del sector confección.

\section{Figura 12}

\section{Marco jurídico como obstáculo a la inversión privada}

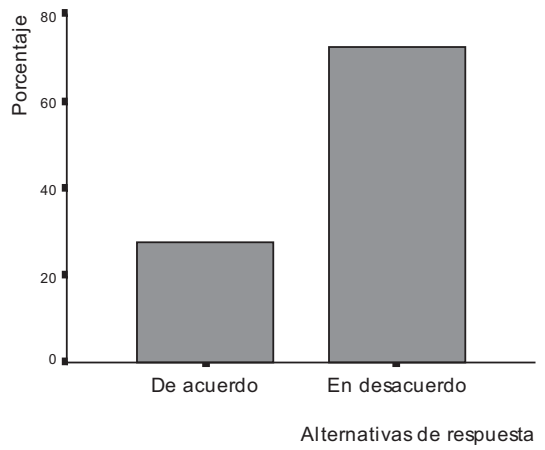

Fuente: Sandrea, 2005. Con base a la entrevista realizada a empresarios del sector confección.

complejo y costoso, a pesar de que la constitución garantiza la gratuidad de la justicia, esto sólo se refiere a la exoneración del pago de aranceles judiciales, pero han quedado otros costos tales como: renombre de los abogados, especialización, tiempo, entre otros. El estudio realizado por CONAPRI en el 2002 (citado por Malavé, 2002) permitió constatar que la mayor parte de los empresarios consideran en mayor o menor grado que el sistema legal garantiza los contratos y derechos de propiedad en litigios comerciales, sin embargo pocas empresas recurren al mismo para la solución de conflictos.

Resultados similares a los obtenidos por el CONAPRI se encontraron en la entrevista realizada a los empresarios del sector confección zuliano, quienes consideran que el funcionamiento del marco jurídico no afecta las decisiones de inversión en sus empresas, así un 72,22\% estuvo en desacuerdo con la afirmación de 
que la actuación del sistema de derecho mercantil venezolano constituya un obstáculo para la inversión, por el contrario, algunos manifestaron que muy pocas veces habían tenido que recurrir a éste para resolver un problema en la empresa, por lo que aunque los procesos sean lentos, su actuación no es relevante en las organizaciones (Ver Figura 12).

\section{Conclusiones}

En el sector confección zuliano, el proceso de desinversión registrado a nivel nacional se ha traducido en la reducción del tamaño de las empresas, cuando no en el cierre, también muchas organizaciones han cambiado de nombre por razones de carácter financiero (declaración de quiebra). El número de trabajadores fijos es muy reducido en cada empresas, aunque varía en forma importante a lo largo del año.

Entre los factores técnico-operativos determinantes de la inversión privada tomados en cuenta por los gerentes zulianos destaca: la actualización tecnológica, especialmente en relación a la competencia local. Asimismo consideraron como factores que favorecen las decisiones de inversión la ubicación geográfica del estado Zulia (cercanía de puertos aeropuertos y mercado textil colombiano), el funcionamiento de servicios públicos y la dotación de infraestructura, mientras que como factores desfavorables pueden mencionarse el costo de los servicios públicos, los servicios aduanales y la actuación de las cámaras u otras asociaciones de apoyo a las empresas. Por otro lado, las opiniones estuvieron divididas en cuanto a la incidencia de la inseguridad fí- sica de personas y bienes sobre la motivación a invertir.

A pesar de la opinión favorable de la mayoría de los entrevistados sobre la relación adelantos tecnológicos e inversión, la intención de incorporar tales innovaciones no se materializa en la realidad por lo que la situación económicapolítica y la falta de apoyo institucional constituyen factores de mayor relevancia para las decisiones de inversión privada en el sector.

Con relación al factor político, el $55,56 \%$ de los dueños-gerentes de las empresas del sector confección zuliano consideró que la situación política venezolana desmotiva su deseo de invertir, refiriéndose principalmente a las condiciones generales de incertidumbre y desconfianza en la política venezolana. Mientras que, en cuanto al funcionamiento del marco jurídico, $72,22 \%$ de los entrevistados estuvo en desacuerdo con la afirmación de que la actuación del sistema de derecho mercantil venezolano constituya un obstáculo para las decisiones de inversión. A pesar de que la mayor parte de los entrevistados afirmaron que la incertidumbre y la desconfianza en el ámbito político desmotivaba su deseo de invertir, al mismo tiempo éstos consideraron con mayor influencia el impacto que los factores económicos-financieros reportaron sobre sus decisiones de inversión.

El estímulo a la inversión privada debería incluir programas de actualización tecnológica, financiamiento, apoyo técnico y formación de recursos humanos. Asimismo, la concientización del rol de los empresarios como líderes y/o emprendedores en el destino económico de la nación, en este sentido, es importante 
Factores técnico-operativos y políticos determinantes de las decisiones de inversión Sandrea, Maryana; Boscán, Mariby; Romero, Jenny y Acosta, Ana

modificar la visión del negocio así como sus expectativas sobre lo que puede esperar del Estado venezolano.

Por otra parte, las cámaras y otras agrupaciones privadas de apoyo a las empresas deben redimensionar su actividad, buscando un mayor acercamiento con los empresarios, ya que según el estudio realizado la mayoría de los entrevistados no están afiliados a las cámaras existentes en la región porque consideran que no ofrecen incentivos significativos. Por tanto, es necesario que este tipo de agrupaciones brinde servicios e información realmente valiosas para los industriales especialmente en cuanto a las alianzas con proveedores del sector confección de forma de aplicar estrategias que permitan reducir los costos operativos de las empresas.

\section{Referencias Bibliográficas}

Baptista, Asdrúbal (1997). Teoría Económica del Capitalismo Rentístico. Economía Petróleo y Renta. Ediciones IESA, Caracas, Venezuela.

Baptista, Asdrúbal (2004). El relevo del capitalismo rentístico. Hacia un nuevo Balance de Poder. Fundación Polar, Caracas, Venezuela.

Banco Central de Venezuela (2000-2001). Anuario de Cuentas Nacionales. Colección estadística. Años 20002001. BCV, Caracas, Venezuela.

Banco Mundial BM (2003). Informe sobre el desarrollo económico mundial 20002001. Disponible en http:// www.worIdbank.org; 1-6. Consultado el 12-022003.

Cohen, Aarón y Sabal, Jaime (1988). “¿Por qué invierten los inversionistas?”. Los determinantes de la inversión privada.
En Las empresas venezolanas su gerencia. Compilador/ Director del proyecto: Moisés Naim. Ediciones IESA, C.A., Caracas, Venezuela.

García, Angel y Plaza, Shakyra (2000). Implicaciones del contexto inflacionario sobre la inversión en el sector manufacturero venezolano. Revista Venezolana de Análisis de Coyuntura. Vol. VI. No. 2: 281-308.

Instituto de Investigaciones (2004). Base de datos programa "Factores de competitividad de las Pequeñas y Medianas empresas manufactureras en la región zuliana”. Maracaibo, Venezuela. Mimeo.

Malavé, José (2002). Todo lo que usted quería saber sobre... Costos y obstáculos a los negocios: La opinión de empresarios y gerentes. Sección de la revista Debates IESA, Volumen VIII No 1: 43-52.

McConnell, Campbell y Brue, Stanley (2001). Economía. McGraw-Hill Interamericana Colombia.

Morales, Mariher, Romero Jenny, Andrade, Nancy (2002). Competitividad de la industria de la confección de Municipio Maracaibo-Estado Zulia. Revista Ciencias Sociales (RCS). Vol. VIII, N0. 3: 464-485.

Monteferrante, Patricia (2002). Los negocios: los otros dolientes de la inseguridad. Revista "Debates IESA". Volumen VIII No. 1: 20-24.

Parra, Gastón (2001). Condiciones y perspectivas del crecimiento económico en Venezuela. Revista Banco Central de Venezuela (BCV) Foros. No.6: 25-38.

Penfold, Michael (2002). ¿Cuánto cuesta invertir en Venezuela?. Revista "Debates IESA". Volumen VIII No. 1: 8-11.

Porter, Michael (1991). La Ventaja Competitiva de las Naciones. Javier Vergara Editor S.A., Buenos Aires, Argentina. 
Romero, Jenny, Sandrea, Maryana, Morales, Mariher, Boscán, Mariby, Acosta, Ana (2000). La industria de la confección Zuliana en la era de la competitividad. Revista Venezolana de Gerencia. Año 5 No. 11: 189-208.

República Bolivariana de Venezuela (2001). Decreto Ley para la Promoción y Desarrollo de la Pequeña y Mediana Industria. Gaceta Oficial No. 5.552 extraordinario: 50-57.

Sandrea, Maryana y Boscán, Mariby (2004). La cadena de valor del sector confección. Revista Venezolana de Gerencia. Año 9 No. 26: 336-356.
Sandrea, Maryana (2005). Factores determinantes de la inversión privada en las pequeñas y medianas empresas del sector confección zuliano. Trabajo de grado para optar al título de Magíster en Gerencia de Empresa. Universidad del Zulia. Maracaibo, Venezuela.

Shatz, Howard (2001). Expandiendo la inversión extranjera directa en los países Andinos. Papel de trabajo No 64: $1-46$.

Singer, Dinorah y Lombardi, Diego (2002). Infraestructura y servicios: ¿obstáculos a la inversión? Revista "Debates IESA", Volumen VIII No. 1: 34-42. 